\title{
Objective Measurement of Health Pigs - Application of acute Phase Proteins
}

\author{
By Jens Peter Nielsen and Henrik Hagbard Petersen \\ The Royal Veterinary and Agricultural University, Copenhagen, Denmark.
}

In this presentation health is defined as absence of disease in individual animals or populations. High health status indicates that no or only mild disease is present. Low health status indicates that several or severe diseases are present.

Measurement of health status. Objective measurement of health status is difficult. Serological or microbiological testing for a number of specific infections is possible, but often impractical. Clinical examination may reveal clinical disease problems but do not reveal sub-clinical disease an may suffer from lack of objectivity. Sub-clinical disease may be measured indirectly by recording of growth rate, feed consumption or reproductive performance. However, these indicators are difficult to interpret. Pathological findings at slaughter only give a prevalence estimate and data-quality may be poor. Acute phase proteins are produced as a non-specific response to inflammation, trauma and infection in animals and may be measured objectively in serum samples.

Why measure health status? Objective measurement of health status or disease severity is important in several situations. It creates an important research tool when studying pathogenesis and inflammatory processes in experimental animals or when testing the efficacy of pharmaceuticals or comparing their effect in experimental or field trials. Objective measurement of health status allows comparison of health in different production. Categorisation of herds in health levels may be relevant in the future.

Acute phase proteins in pigs. Acute phase proteins are an important part of the acute phase response following tissue damages. The damage may be induced by infections, toxins, trauma etc. and results in liberation of inflammatory mediators into the systemic circulation. These mediators include cytokines such as IL-1, TNF, IL-4, IL-6, IL-8, IL-10 and IL-11. The cytokines induce increased (positive) or decreased (negative) hepatic synthesis of serum acute phase proteins. Several porcine infections has been shown to mount an acute phase response e.g. Actinobacillus pleuropneumoniae, toxigenic Pasteurella multocida, Mycoplasma hyorhinis, PRRS virus etc. The major acute phase proteins in pigs include haptoglobin, major acute phase protein (MAA), serum amyloid A (SAA), alfa1 acid glycoprotein (AGP) and C-reactive protein.

Serum haptoglobin in relation to clinical signs and production systems. In a case-control study in 14 pig herds including serum samples from 170 healthy and clinically diseased pig pairs. A significant increase in haptoglobin concentrations was found in pigs with clinical signs such as respiratory disease, lameness, ear and tail biting and diarrhoea. In another study 7 herds free from 5 specific pathogens (Danish SPF-herds) were compared to 4 conventional herds suffering from some or all of the infections absent in the SPF herds. In each herd serum samples were taken from young, medium or old finishing pigs. At the same time the animals were subjected to a standardised clinical examination. The study showed that finishing pigs from SPF herds had a significantly lower haptoglobin serum concentration than pigs from conventional herds. However, the variation between SPF herds was high, indicating that SPF-production is not always equivalent to high health status. 